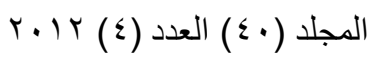 \\ تأثير موعد وشدة التقليم في بعض خواص النمو والحاصل لأشجار المشمش صنف زيني

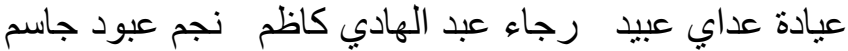 \\ قسم البستنة و هندسة الحدائق / كلية الزر اعة / جامعة بأب الغداد
}

\section{الخلاصة}

أجريت هذه الدر اسة في بستان المشمش التابع لقسم البستنة و هندسة الحدائق / كلية الزراعة / جامعة

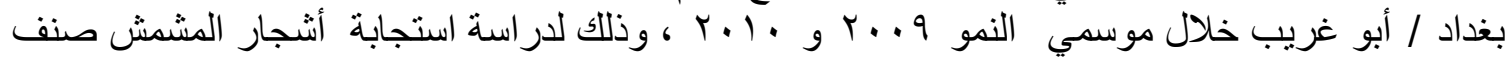

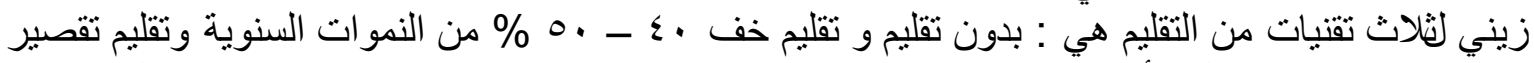

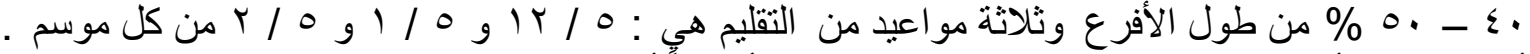

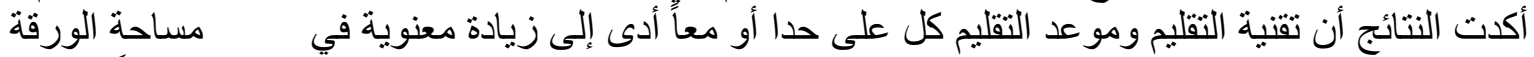

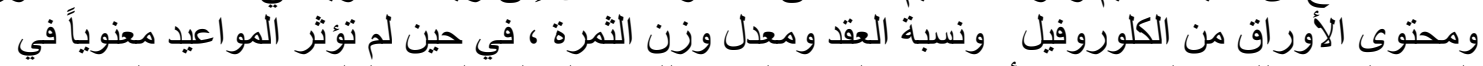

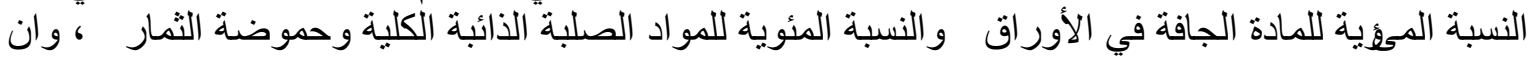

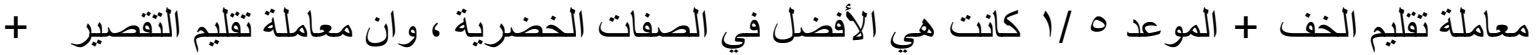

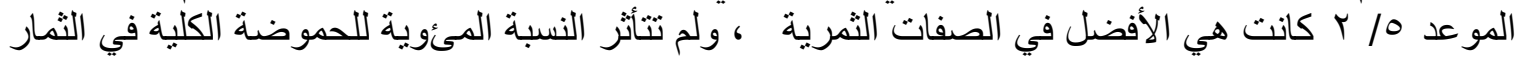
بمعاملات التقليم المختلفة

\section{المقدمة}

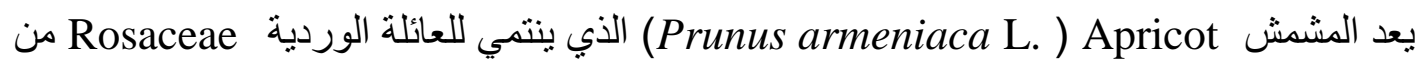

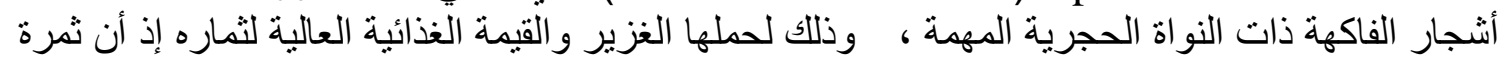

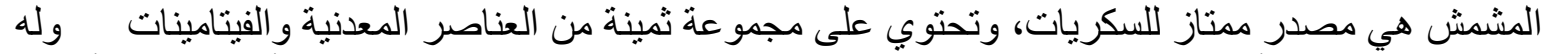

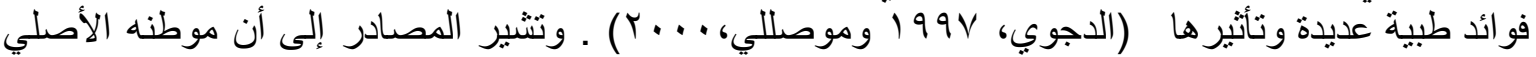

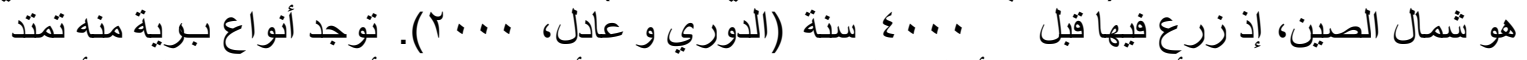

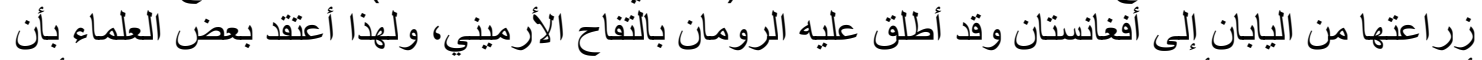

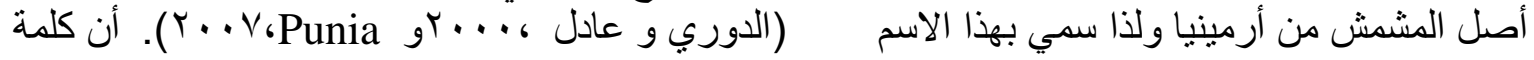

AL-Praecox Apricot

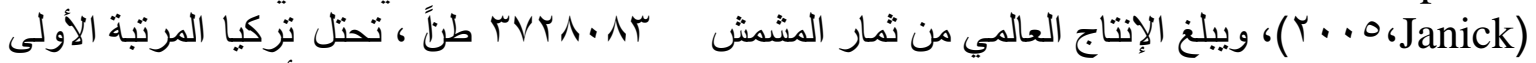

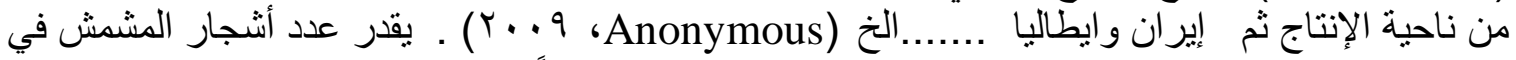

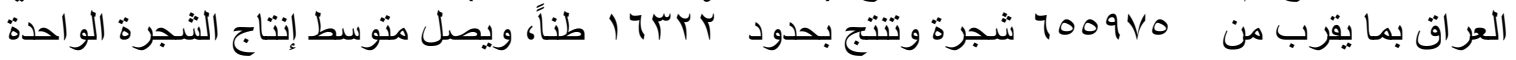

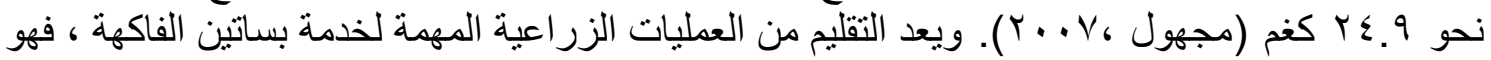

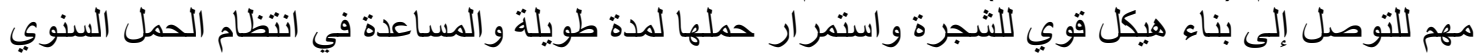

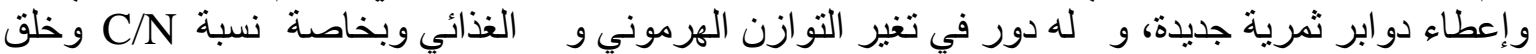

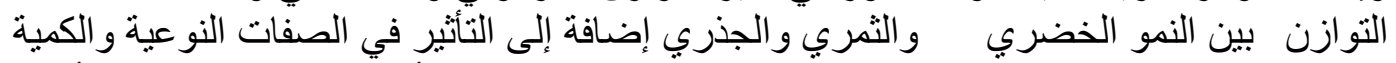

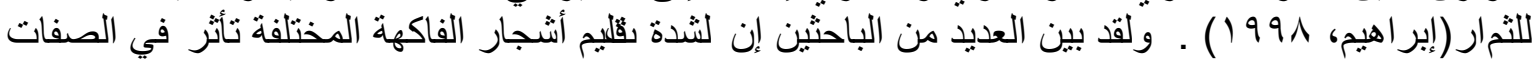

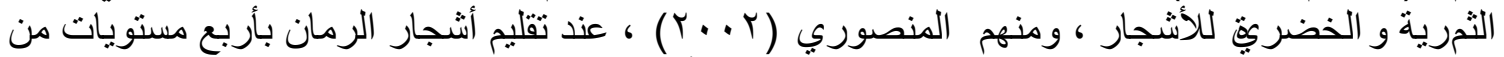

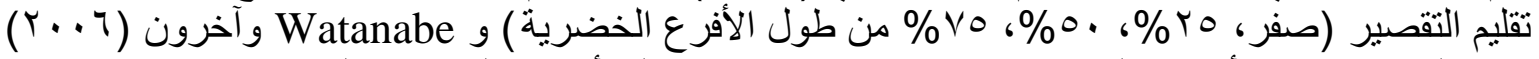

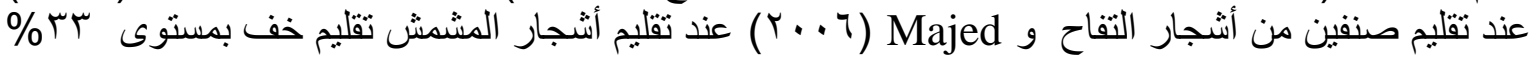

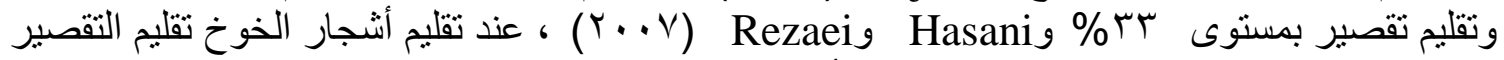

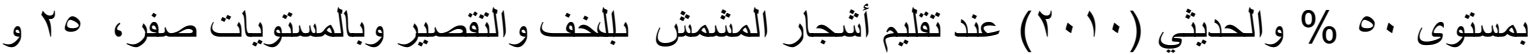

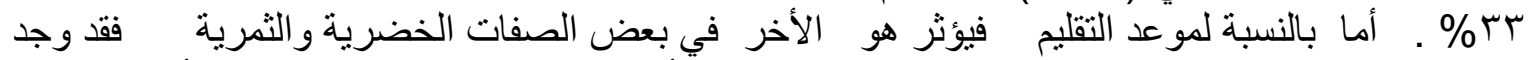

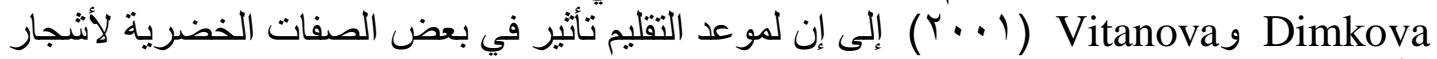

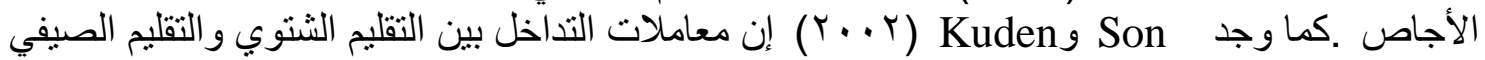

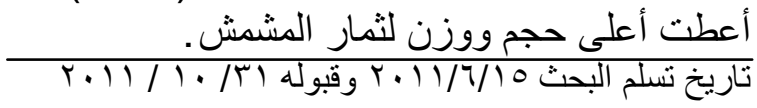

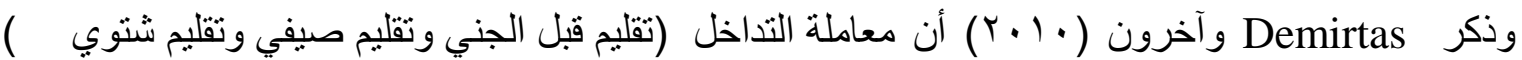
لأشجار المشمش أدى إلى زيادة في حاصل الأشجار وتحسين صفات النمو الخضري للأشجار في حين 


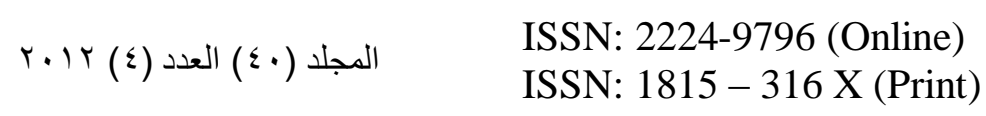$$
\text { مجلة زر اعة الر افدين }
$$

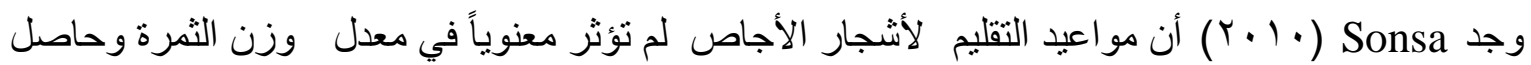

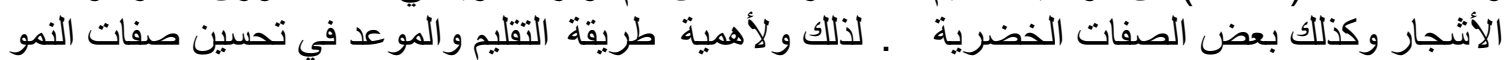

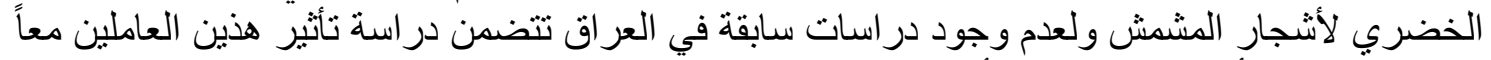

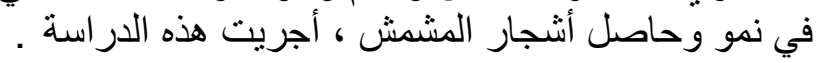

\section{مواد الرحث وطرائقه}

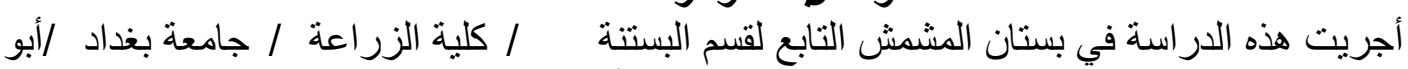

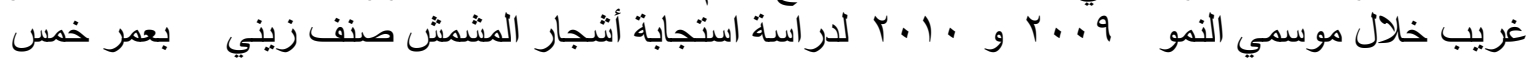

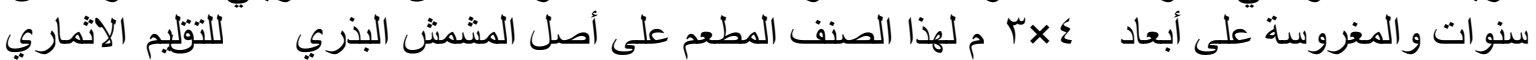

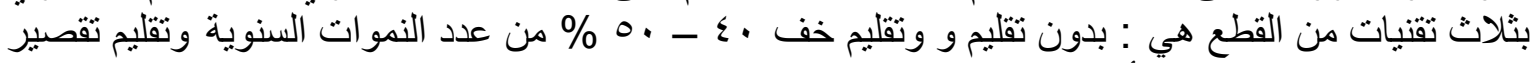

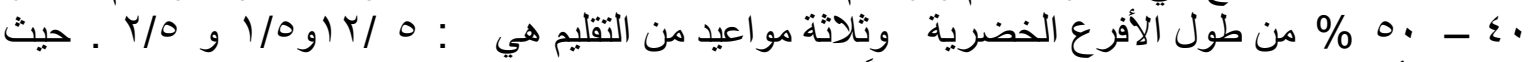

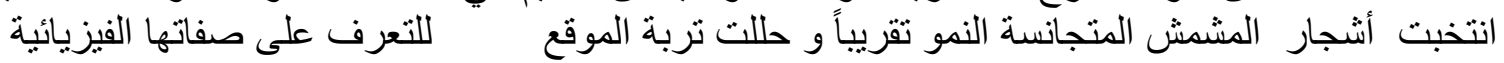

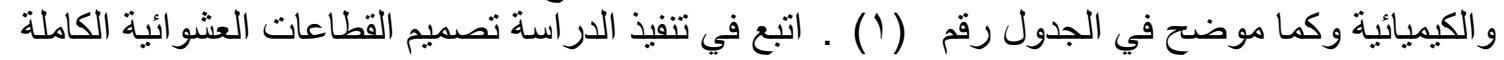
و RCBD

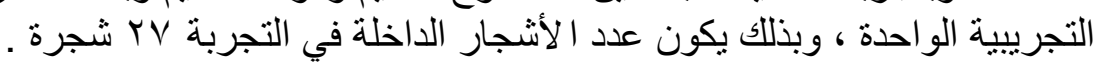

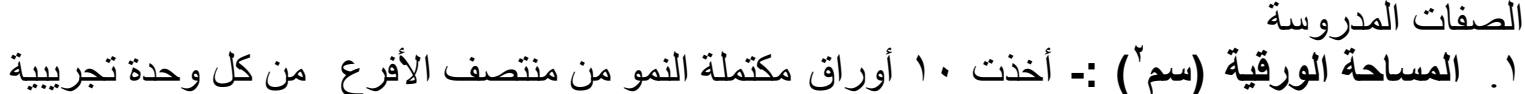

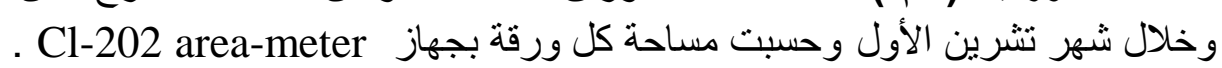

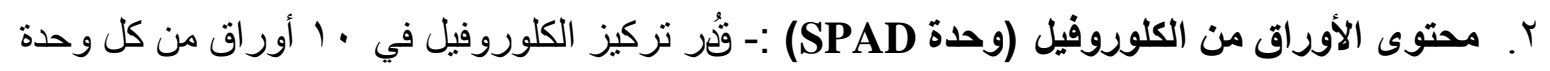

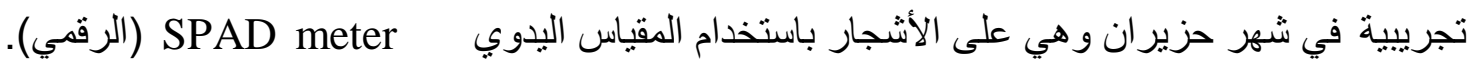
(r.... Nina Felixloh)

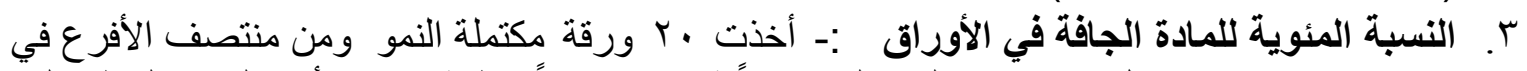

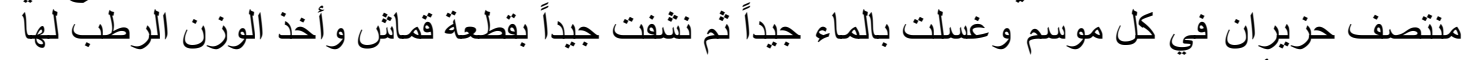

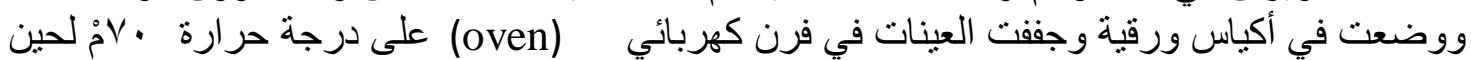

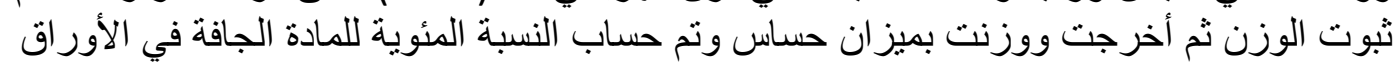
كالآتي

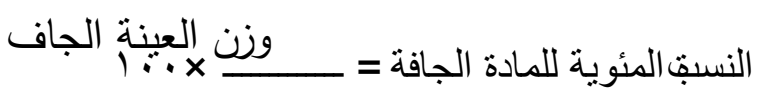

$$
\begin{aligned}
& \text { وفق ما جاء في (الصحاف،919 ( ). الوزن الطري }
\end{aligned}
$$

ع. نسبة العقد :- استخرجت هذه النسبة بحساب عدد الأزهار في فرع هيكلي كامل في مرحلة التزهير

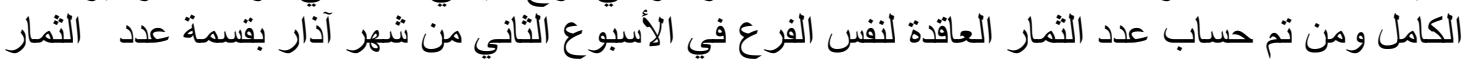

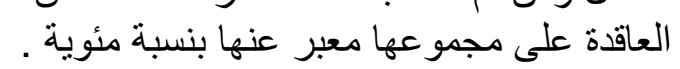

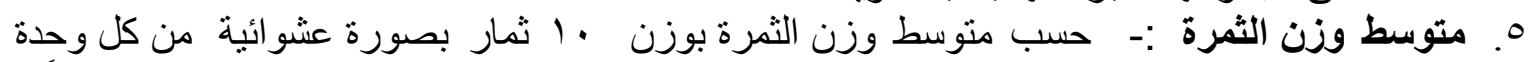

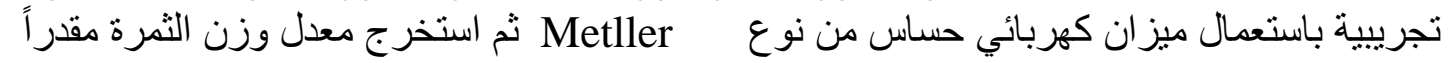
7. النسبة المئوية للمواد الصلبة الذائبة الكلية :- قيست النسبة المئوية للمواد الصلبة الذائبة الكلية في

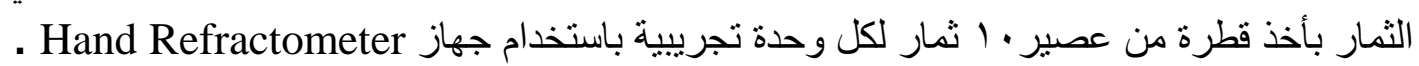

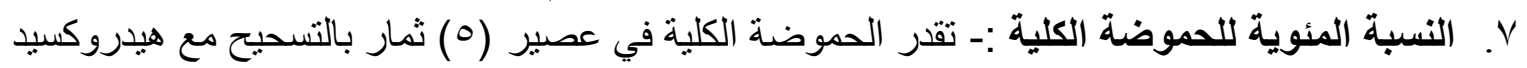

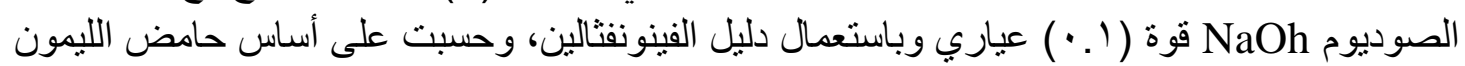

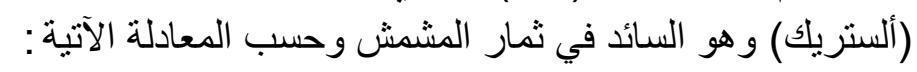




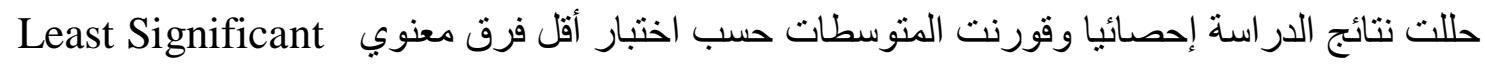
(L.S.D) Difference

\begin{tabular}{|c|c|}
\hline القيمة & الصفة \\
\hline غرينية طينية & نسجة التربة \\
\hline 11 & الرمل \% \\
\hline$\leqslant 7$ & الغرين \% \\
\hline r4 & الطين ال \\
\hline 8.77 & $\mathrm{pH}$ \\
\hline י & النتروجين (\%)الجاهز \\
\hline$\cdot g \cdot r$ & الفسفور (\%) الجاهز \\
\hline$\cdot g V 7 T$ & البوتاسيوم (ملغم / لتر) الجاهز \\
\hline$\varepsilon g \leqslant 0$ & الكلور (ملغم / لتر) \\
\hline$\vee g \wedge \varepsilon$ & الصوديوم (ملغم / لتر) \\
\hline $7,1 \varepsilon$ & المغنيسيوم (ملغم / لتر ) \\
\hline 9,70 & الكالسيوم (ملغم / لتر) \\
\hline rgor & (cm/mmohs) EC \\
\hline
\end{tabular}

\section{النتائج والمناقشة

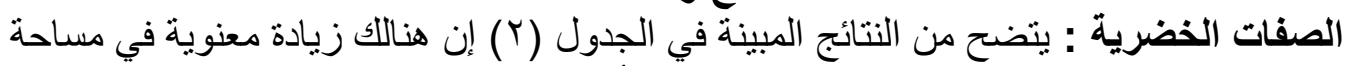

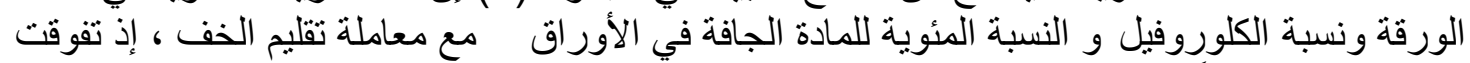

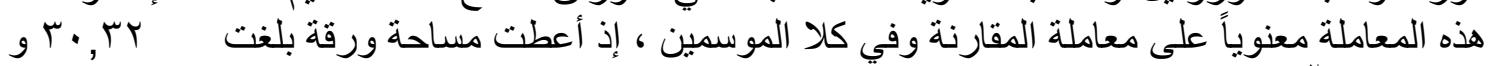

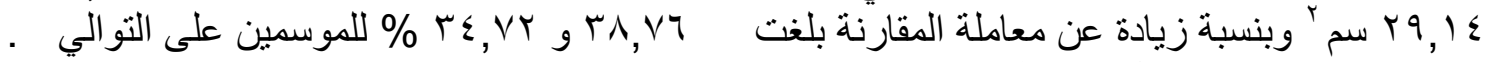

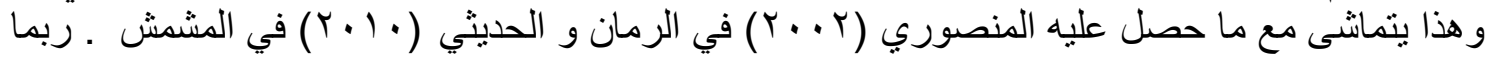

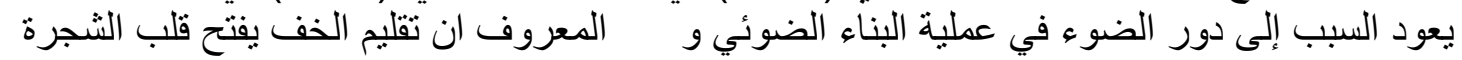

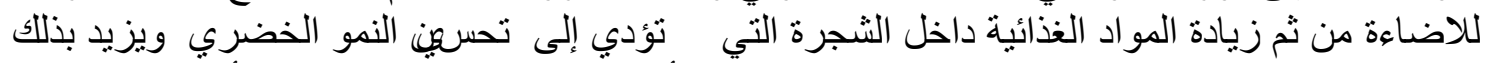

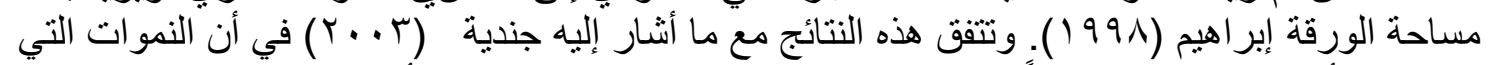

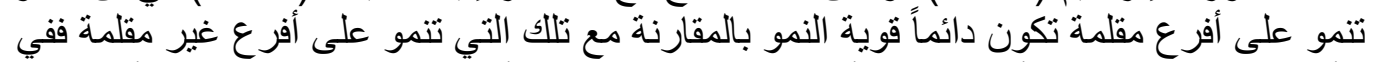

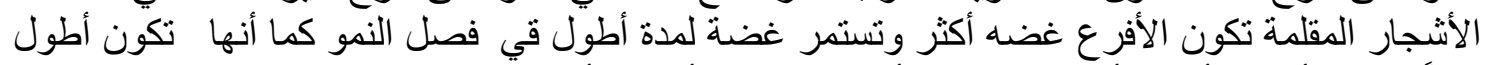

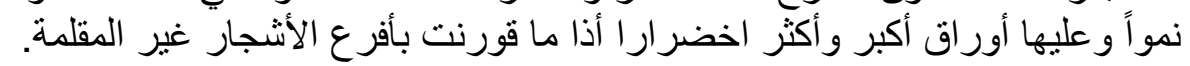

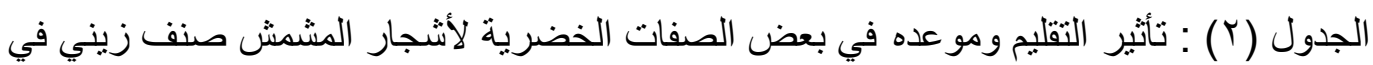

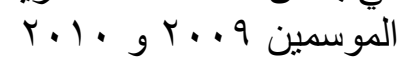

\begin{tabular}{|c|c|c|}
\hline$r .1$. & $r \ldots q$ & الموسم \\
\hline مو عد التقليم & مو عد التقليم & تقنية \\
\hline
\end{tabular}




\begin{tabular}{|c|c|c|c|c|c|c|c|c|}
\hline المتوسط & r/O & $1 / 0$ & $1 \% / 0$ & المتوسط & $r / 0$ & $1 / 0$ & $14 / 0$ & التقليم \\
\hline \multicolumn{9}{|c|}{ مساحة الورقة (سم') } \\
\hline T1.7r & Tr.OT & YI.IE & $Y \cdot . Y Y$ & YI.10 & TY.TA & YI.0. & TI.ru & بدون \\
\hline r9.1 & Yq. $\leqslant Y$ & $r . .11$ & rV.q. & $r \cdot . r r$ & $r \cdot r \varepsilon$ & TI. $\leqslant r$ & 19.19 & خف \\
\hline \multirow[t]{4}{*}{$r V . \varepsilon r$} & $r \wedge . \leqslant 7$ & YA.YI & ro.7r & $r V .10$ & rA. $\cdot T$ & TV.10 & rT.YT & تقصير \\
\hline & $r V . I T$ & rq. $\leqslant q$ & $r \leqslant .01$ & & $r V_{.} \cdot r$ & r7. 79 & ro.09 & الهتوسط \\
\hline & ل للتداخل & للمو عد & للتقنية & & ل للتداخل & للمو عد & للتقنية & \multirow{2}{*}{ L.S.D5\% } \\
\hline & $\varepsilon . \cdots$ & 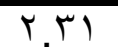 & Y.M & & 4.10 & $1 . r \varepsilon$ & $1 . r \varepsilon$ & \\
\hline \multicolumn{9}{|c|}{ الكلوروفيل (SPAD) } \\
\hline$r 7.1 r$ & $r v . \wedge I$ & Y0.97 & TE.TT & $r \varepsilon .9 V$ & $r 7.91$ & $r \varepsilon .71$ & & بدون \\
\hline$r \cdot . \Sigma \tau$ & $r \cdot .9 \varepsilon$ & (I. & TA.TY & $\Gamma . \cdot r$ & rr. 9 & T.01 & $r V . \leqslant q$ & خف \\
\hline \multirow[t]{4}{*}{$r V .11$} & 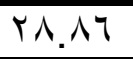 & TV.OT & Y0.1 & 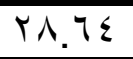 & r9.0Y & $r \cdot . Y T$ & r7.11 & تقصير \\
\hline & rq. . . & YA.OT & $r 7 . \cdot r$ & & r9.01 & Yq. $\leqslant V$ & ro.77 & المتوسط \\
\hline & ل للتداخل & للمو عد & للتقنية & & ل للتداخل & للمو عد & للتقنية & \multirow[t]{2}{*}{ L.S.D5\% } \\
\hline & $0 . \cdot \varepsilon$ & r.91 & r.91 & & $0.9 r$ & $r . \leqslant Y$ & $r . \Sigma Y$ & \\
\hline \multicolumn{9}{|c|}{ نسبة المادة الجافة في الاور اق (\%) } \\
\hline$r 9.1 r$ & rА. TV & rq.Av & ґ.А૫ & $\varepsilon . .1 \varepsilon$ & $\leqslant 1 . \cdot 1$ & rV.T & $\varepsilon 1 . V V$ & بدون \\
\hline$\varepsilon \varepsilon . \wedge \mu$ & $\leqslant 0 . Y V$ & $\{0 . \mu$ & $\varepsilon r .9 r$ & $\varepsilon \varepsilon . \varepsilon$. & $\varepsilon \varepsilon .+r$ & $\leqslant 0.04$ & $\varepsilon r .0$ & خف \\
\hline \multirow[t]{4}{*}{$\varepsilon \varepsilon .0$} & $\leq 0.1 T$ & हT.T० & $\varepsilon r .7 \wedge$ & $\varepsilon r .1$ & $\varepsilon \varepsilon . \cdot V$ & $\varepsilon Y .19$ & $\varepsilon Y . V T$ & تقصير \\
\hline & $\varepsilon r . \cdot r$ & $\varepsilon Y . \wedge \varepsilon$ & $\varepsilon Y .10$ & & $\varepsilon r . \cdot \varepsilon$ & $\varepsilon 1 . \vee \wedge$ & $\varepsilon Y .0 T$ & المتوسط \\
\hline & للتداخل & للمو عد & للتقنية & & للتداخل & للمو عد & للتقنية & \multirow[t]{2}{*}{ L.S.D5\% } \\
\hline & $\varepsilon . Y$ & N.S & $r . \varepsilon r$ & & $r . r$ & $1.1 \mathrm{~V}$ & $1.1 \mathrm{~V}$ & \\
\hline
\end{tabular}

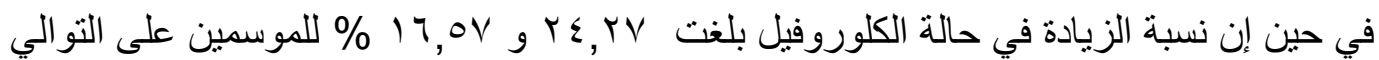

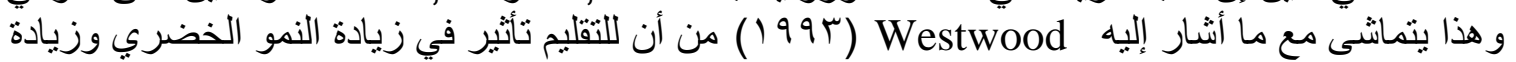

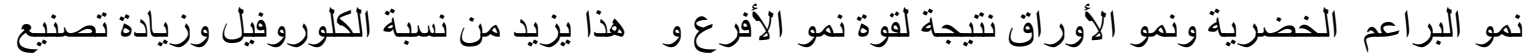

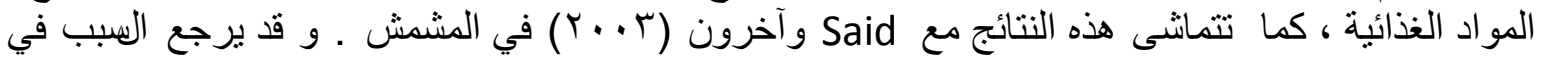

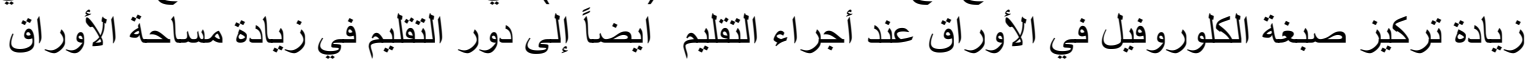

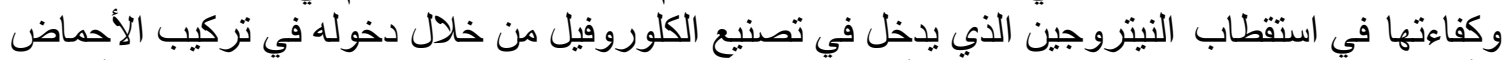

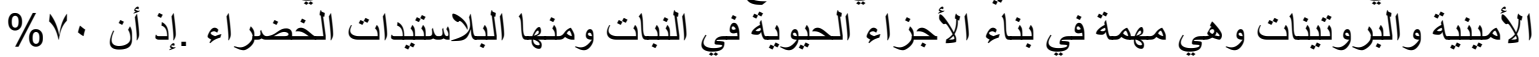

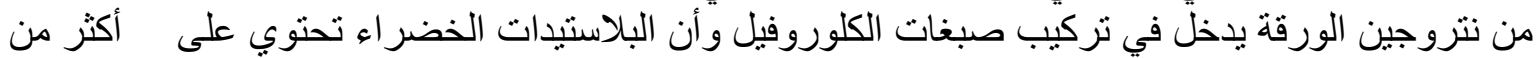

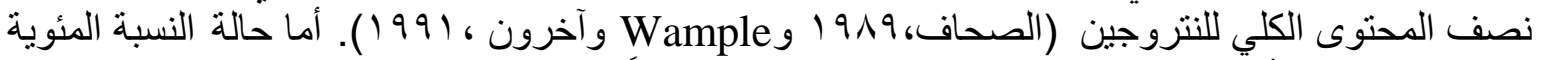

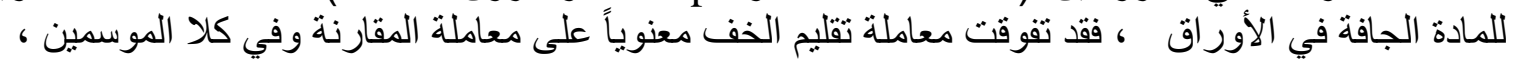

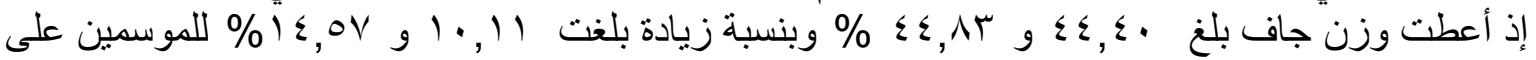

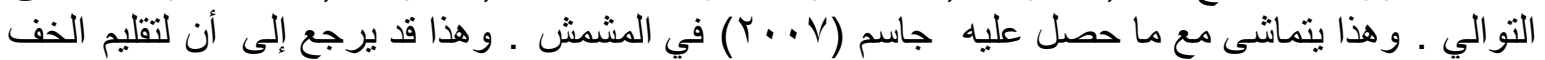

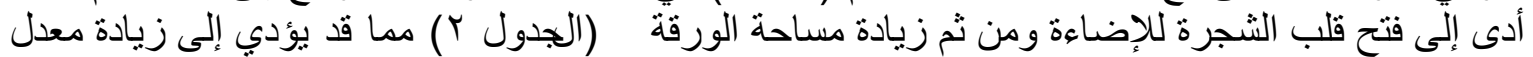

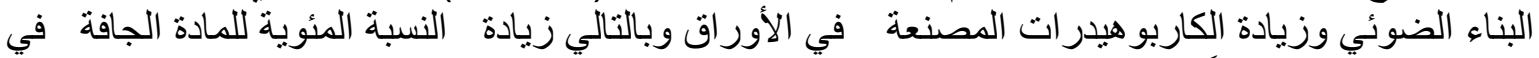

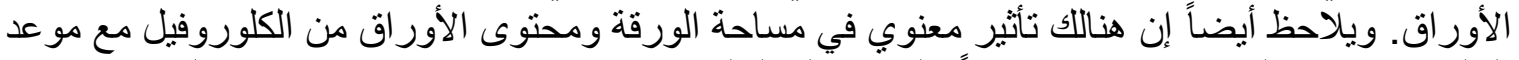

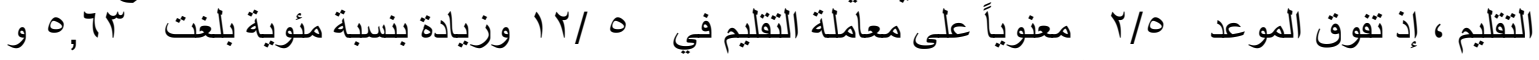

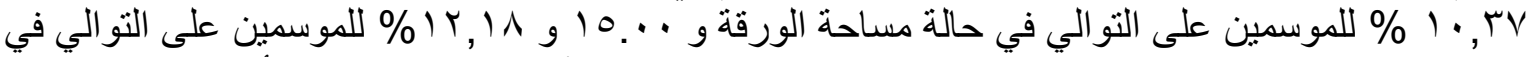

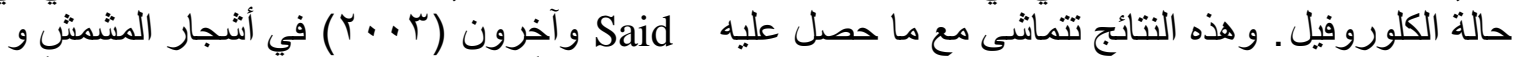

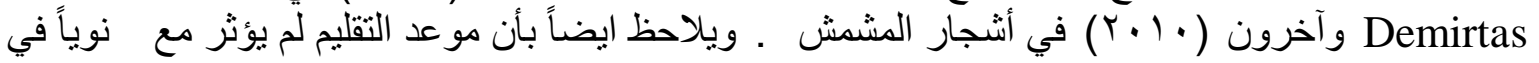




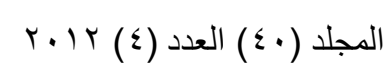

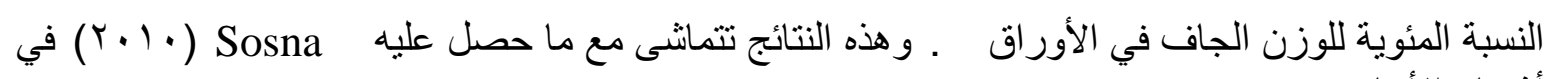

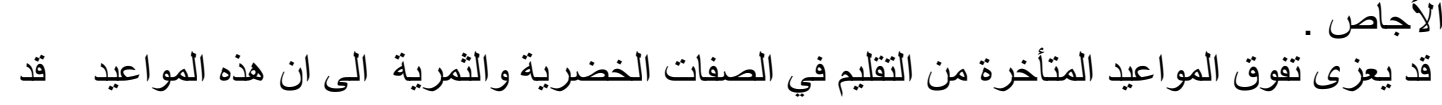

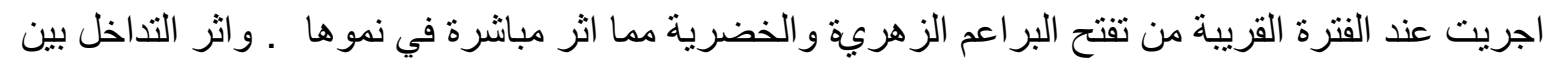

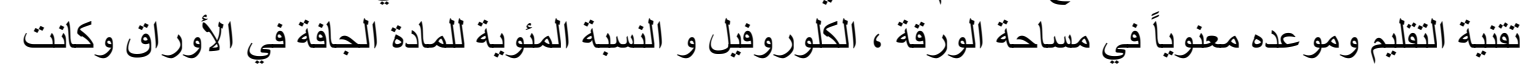

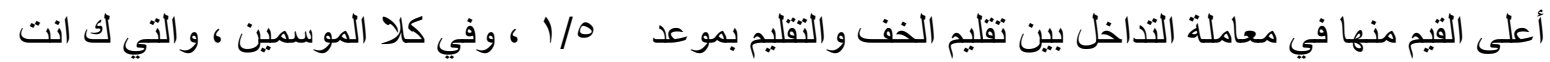

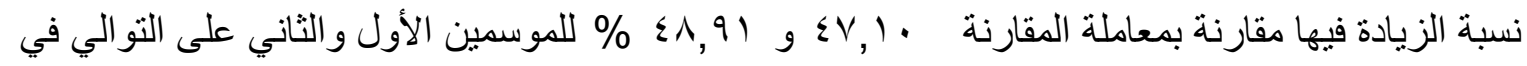

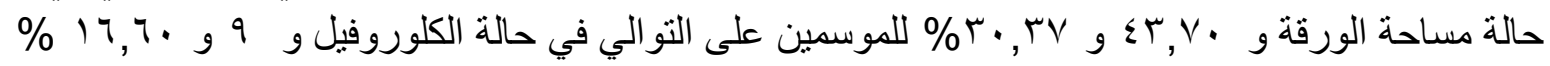

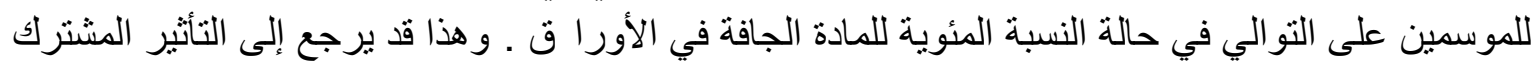

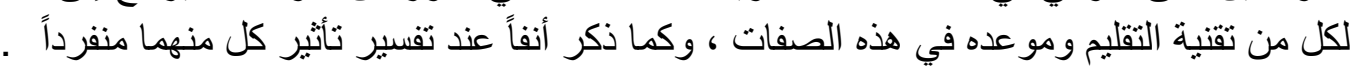

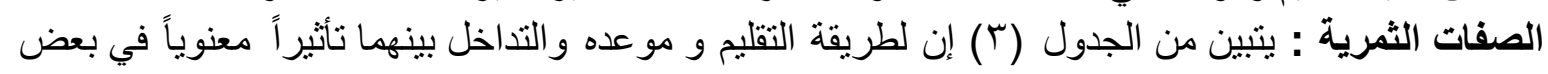

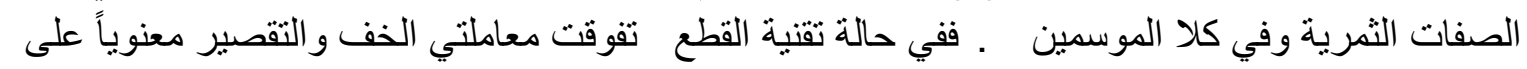

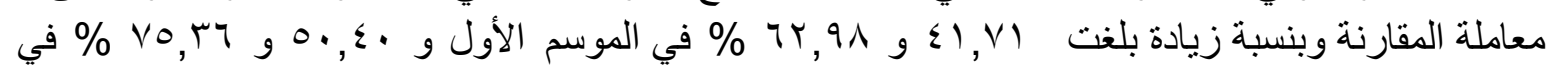

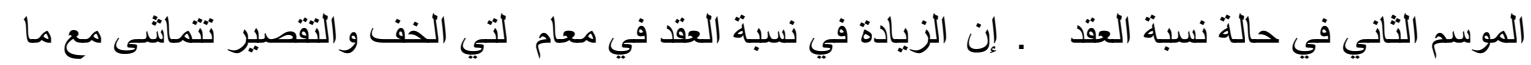

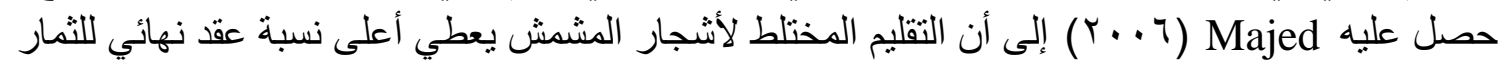

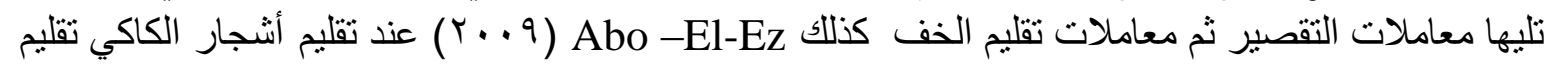

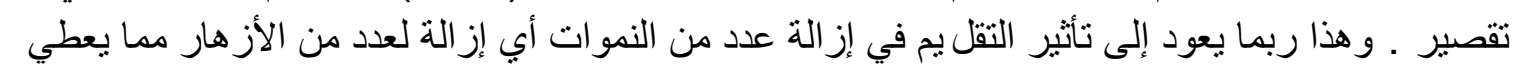

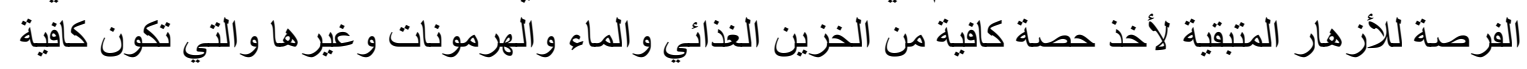

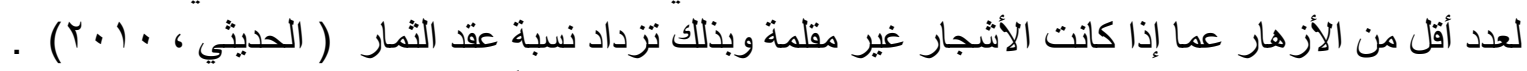

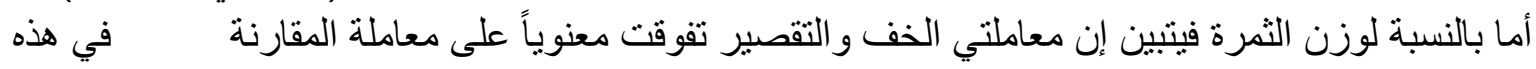

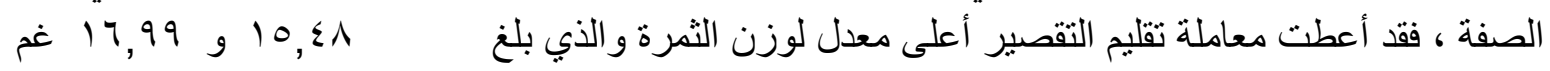

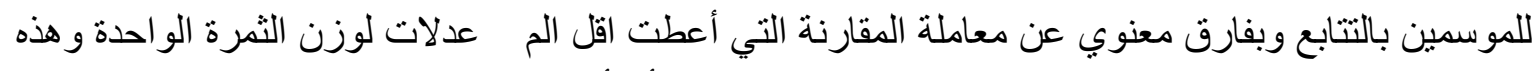

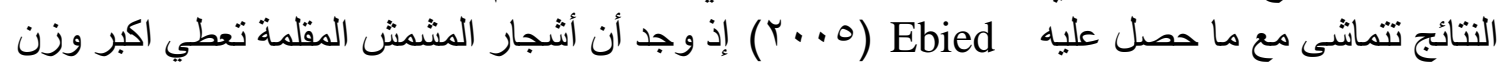

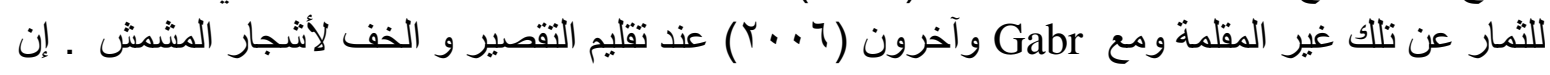

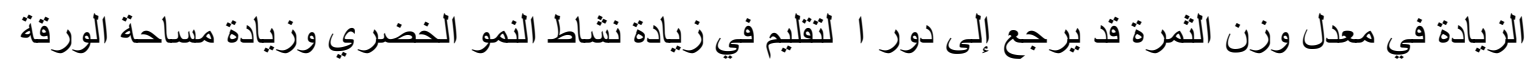

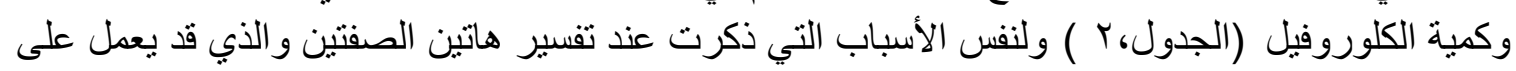

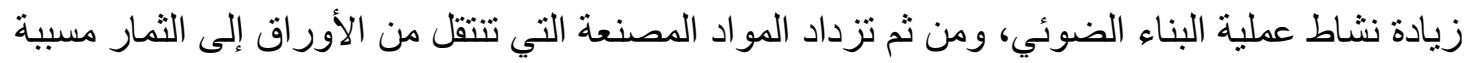

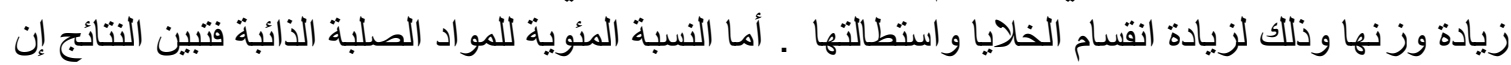

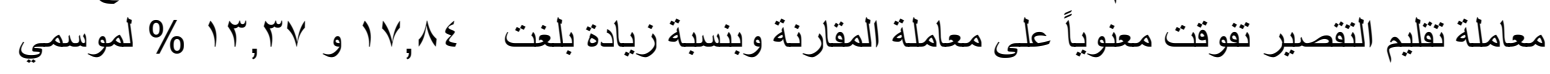

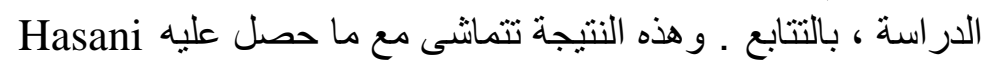

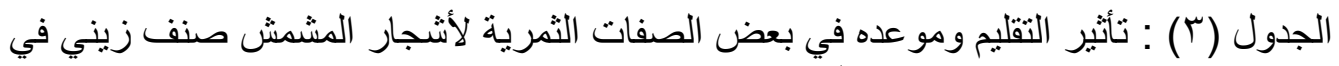

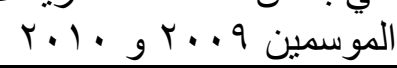

\begin{tabular}{|c|c|c|c|c|c|c|c|c|}
\hline \multicolumn{4}{|c|}{ r.l. } & \multicolumn{4}{|c|}{$r \ldots q$} & الموسم \\
\hline \multicolumn{4}{|c|}{ مو عد التقليم } & \multicolumn{4}{|c|}{ مو عد التقليم } & \multirow{2}{*}{ التقليم } \\
\hline المتوسط & $r / 0$ & $1 / 0$ & $T / 10$ & المتوسط & $r / 0$ & $1 / 0$ & $1 \pi / 0$ & \\
\hline \multicolumn{9}{|c|}{ نسبة العقد (\%) } \\
\hline 7.19 & $\nabla . \wedge \mu$ & $0.9 \leqslant$ & 0.11 & $0.9 \mathrm{~V}$ & $\Lambda .11$ & $0 . \leqslant \varepsilon$ & $\varepsilon . \Upsilon_{q}$ & بدون تقليم \\
\hline $9 . \leqslant 7$ & $11 . \cdot v$ & Q.AY & $V . \sum \Lambda$ & ᄉ. $\leqslant 7$ & $9 . \varepsilon r$ & 9.01 & $7 . \mu V$ & تقليم خف \\
\hline \multirow[t]{2}{*}{11.4} & $T Y .1 Y$ & $11.0 Y$ & $9 . \varepsilon \%$ & $9 . \mathrm{Vr}$ & $11 . \mathrm{NV}$ & $1 . .14$ & 8.19 & تقلبي تقصير \\
\hline & $1 \cdot r \varepsilon$ & 9.99 & $\overline{V . \mu \varepsilon}$ & & $9 . \wedge r$ & $\Lambda . \Gamma \Lambda$ & 0.90 & المتوسط \\
\hline
\end{tabular}




\begin{tabular}{|c|c|c|c|c|c|c|c|c|}
\hline & للتثاخل & للمو عد & للتقنية & & للتنداخل & للمو عد & لللتقنية & \multirow{2}{*}{ L.S.D5\% } \\
\hline & $r .04$ & $r_{.} \cdot \varepsilon$ & $r . \varepsilon$ & & $r .1$. & 1.89 & 1.19 & \\
\hline \multicolumn{9}{|c|}{ وزن الثمرة (غم) } \\
\hline 14.91 & $1 \varepsilon .99$ & $1 r . \leqslant$ & 11.41 & $|Y . Y|$ & 11.99 & $1 Y . \leqslant V$ & $1 \cdot .11$ & بدون تقليم \\
\hline 10.87 & $17.0 \mathrm{~V}$ & $17 . \wedge r$ & $1 \pi . \Lambda \Lambda$ & $1 \leqslant .80$ & $17 . \mathrm{KA}$ & $1 \leqslant .00$ & $1 T . \varepsilon \pi$ & تقليم خف \\
\hline \multirow[t]{4}{*}{17.99} & $11 . \varepsilon r$ & 18.11 & $10 . \varepsilon r$ & 10.21 & 17.04 & $10.1 \varepsilon$ & $1 \varepsilon .7$ & تقليم تقصير \\
\hline & 17.17 & 10.19 & 11.01 & & 10.7. & $1 \varepsilon .19$ & $1 Y .07$ & المتوسط \\
\hline & للتداخل & للمو عد & للتقنية & & للتداخل & للمو عد & للتقنية & \multirow{2}{*}{ L.S.D5\% } \\
\hline & $r . v$ & $1 . V V$ & $1 . \mathrm{VV}$ & & r.rs & $1 . r 0$ & 1.10 & \\
\hline \multicolumn{9}{|c|}{ (\%) T.S.S } \\
\hline $1 \leq . \wedge 1$ & $10.0 Y$ & 10.11 & $1 \varepsilon \ldots$ & $1 \leq . \% 0$ & $1 \leqslant .17$ & 10.50 & r.r. & بدون تقليم \\
\hline 17.77 & 17.99 & $17 . V Y$ & 17.51 & 10.7. & 17.11 & 10.17 & 10.0 & تقليم خف \\
\hline \multirow[t]{4}{*}{$17.1 \mathrm{~V}$} & 11.4. & $17.0 Y$ & 10.9. & 17.91 & $|V . V|$ & 17.04 & 17.21 & تقليم تقصبر \\
\hline & 17.9. & 17.14 & 10.19 & & 17.0 & 10.90 & $1 \varepsilon .97$ & المتوسط \\
\hline & للتنداخل & للمو عد & للتقنية & & للتنداخل & للمو عد & للتقنية & \multirow{2}{*}{ L.S.D5\% } \\
\hline & r.1. & N.S & 1.19 & & r.r. & N.S & 1.91 & \\
\hline \multicolumn{9}{|c|}{ الحموضة (\%) } \\
\hline $1 . r 0$ & $1 . \leqslant 9$ & 1.14 & $1 . Y$. & $1 . r 0$ & 1.0 & $1 . \leqslant 7$ & 1.9 & بدون تقليم \\
\hline $1 . \leqslant 0$ & $1 . \leqslant 1$ & $1 . \varepsilon 1$ & $1.0 Y$ & 1.51 & $1 . \leqslant 0$ & $1 . \varepsilon$ & 1.19 & تقليم خف \\
\hline \multirow[t]{4}{*}{$1 . \leqslant 0$} & $1 . \varepsilon r$ & 1.0 & $1 . \varepsilon r$ & 1.50 & $1 . \leqslant r$ & $1 . \Sigma Y$ & 1.0. & تقليم تقصير \\
\hline & $1 . \leqslant \varepsilon$ & $1 . \varepsilon Y$ & $1 . \mu \wedge$ & & 1.57 & $1 . \leqslant \mu$ & T & المتوسط \\
\hline & للتداخل & للمو عد & للتقنية & & للتداخل & للمو عد & للتقنية & \multirow{2}{*}{ L.S.D5\% } \\
\hline & N.S & N.S & N.S & & N.S & N.S & N.S & \\
\hline
\end{tabular}

و Rezaei

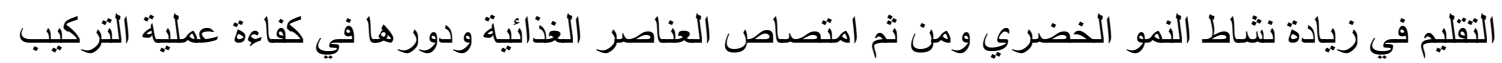

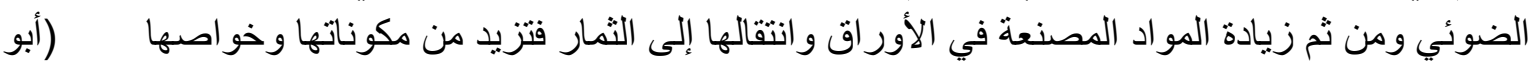

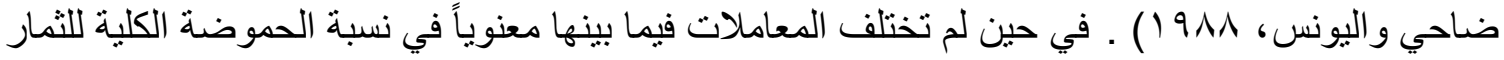

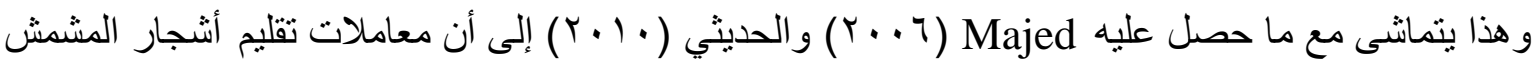

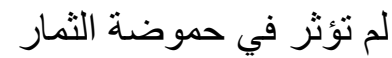

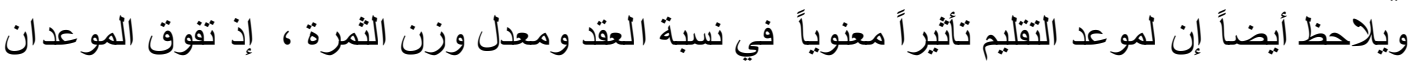

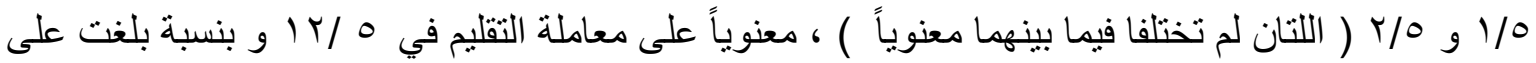

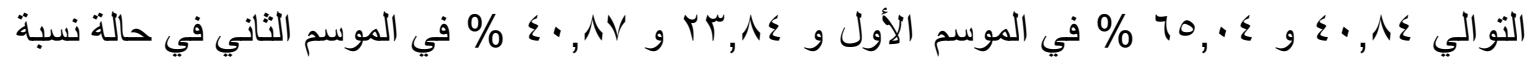

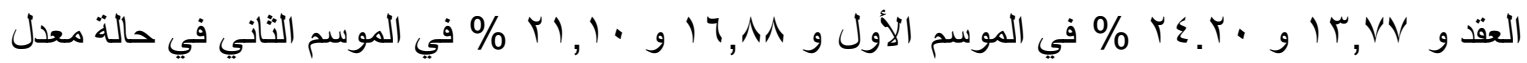

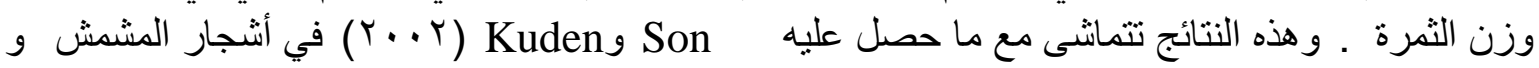

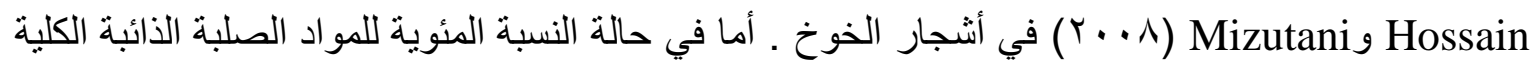

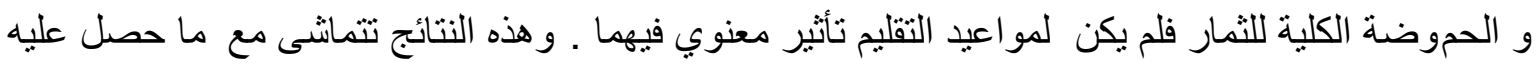
Demirtas

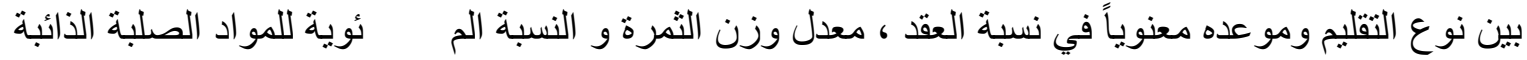

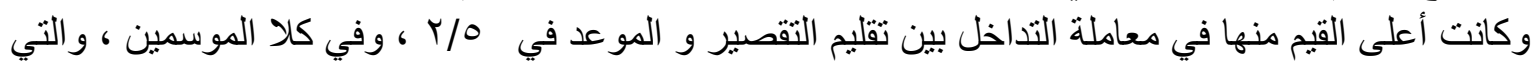




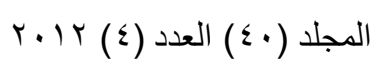
ISSN: 2224-9796 (Online)
ISSN: $1815-316 \mathrm{X}$ (Print)
مجلة زر اعة الر افدين

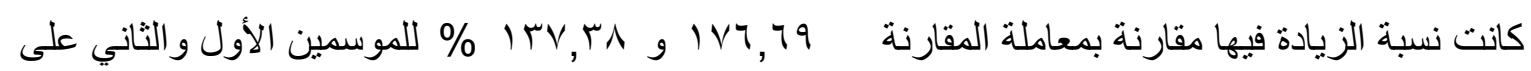

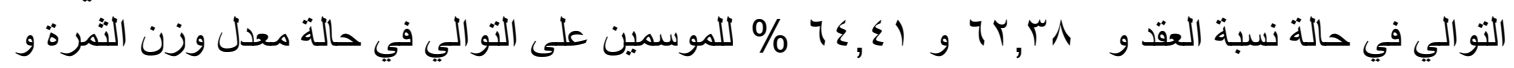

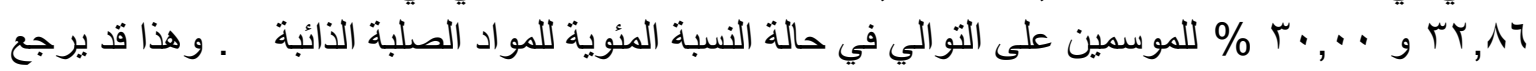
إلى التأثير المشترك لكل من نوع التقليم وموعده في هذه الصفات ، وكما ذكر أنفاً عند تفسير تأثثر كل منهما

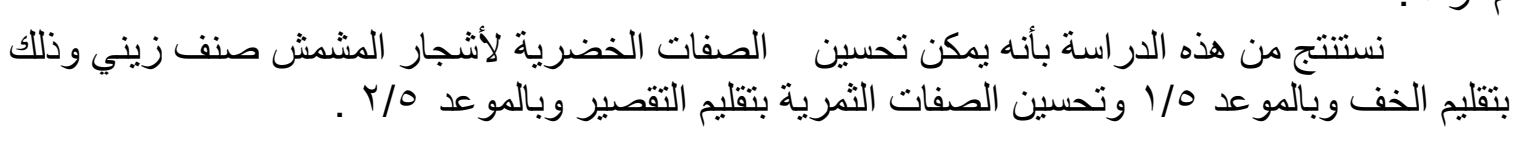

\title{
EFFECT OF PRUNING DATES AND INTENSITY ON SOME GROWTH AND YIELD CHARACTERISTICS OF APRICOT CV. ZAINI
}

Eiada. A. Obaid Rajaa. A. A. Kadhum Najem. A. Jassem Hort. \& Land Scape Dept., Collage of Agric. Baghdad Univ., Iraq

\begin{abstract}
This study was conducted in the apricot orchard of Hort. \& Land Scape Dept./ Collage of Agriculture / University of Baghdad / Abu Ghraib during 2009 and 2010 growing seasons to study the response of apricot trees $\mathrm{cv}$. Zaini to three techniques of pruning ( control, thinning out of $40-50 \%$ from branches number and heading back $40-0 . \%$ from length annually shoots) and time of pruning ( $5 / 12,5 / 1$ and $5 / 2$ of each season). Results showed that techniques of and timing of pruning together or alone aid to significantly effects on leaf area, chlorophyll content, fruit set and fruit weight, whereas the timing were unaffected on dry matter percentage in leaves, T.S.S and acidity. The treatment of thinning out 40$50 \%+5 / 1$ timing was superior in vegetative characteristics, while the treatment heading back of $40-50 \%+5 / 2$ timing was superior with fruit characteristics. Acidity percentage was unaffected with pruning treatments.

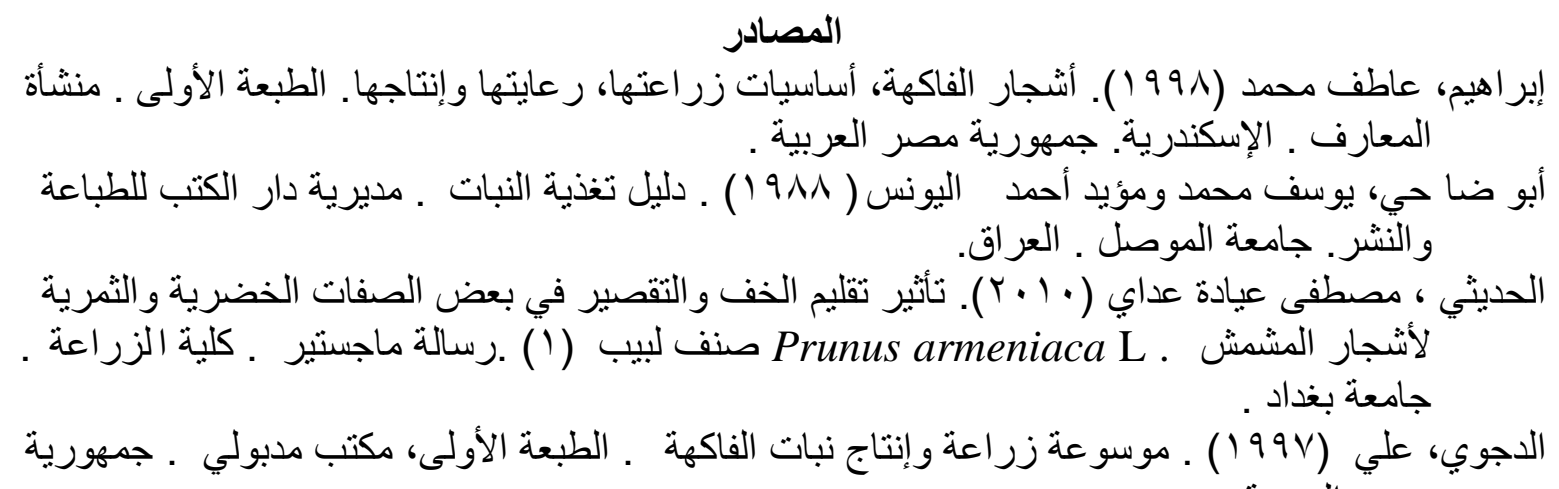

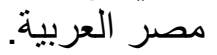

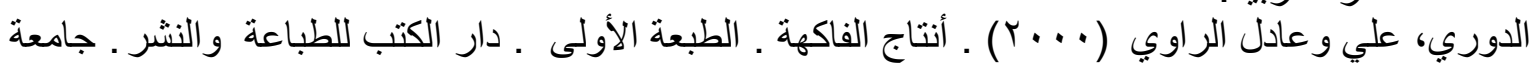

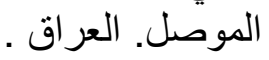

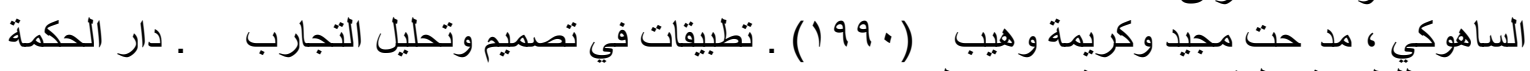

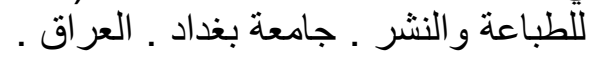

الصحاف، فاضل حسين (919 (19). تغذية النبات التطبيقي. دار الحكمة للطباعة والنشر ـ جامعة بغداد ـ العراق

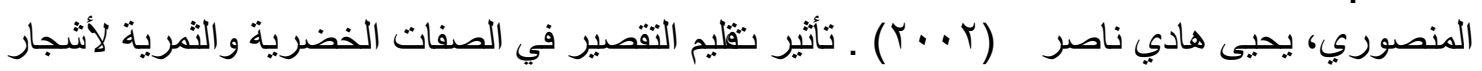

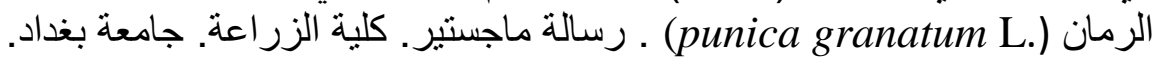




$$
\begin{aligned}
& \text { ～～ISSN: 2224-9796 (Online) } \\
& \text { ISSN: } 1815 \text { - } 316 \text { X (Print) } \\
& \text { مجلة زر اعة الر افدين }
\end{aligned}
$$

Anonymous(2009). Production year book. Rom. F.A.O.www.Faostat.org.

Abo-El-Ez, A.T (2009) . Effect of pruning severity on growth, yield, alternate bearing and fruit quality of "Costata" persimmon (Diospyras Kaki L.) trees . J. Applied Sci. Res. 5(12): 2421-2434.

Demirtas , N. M ; I. Bolat ; S. Ercisli ; A. Ikinci ; H. Olmez ; M. Sahin ; M. Altindag and B. Celik (2010). The effects of different pruning treatments on the growth, fruit quality and yield of 'Hacihaliloglu' apricot . Acta Sci. Pol., Hortorum Cultus. 9(4) : 183-192.

Dimkova, S.D and L.M. Vitanova (2001). Effect of pruning time On the growth and biological uptake of nutrient elements by plum trees. Proceeding of 9th International Conference of Horticulture, September 3th -6th , Czech Republic. 1 (1) :47-51.

Ebied, S.M (2005). Comparative Studies on The Effect of Some Treatments on Flowering and Fruiting in Different Bearing Sites of Canino Apricot Trees . Ph.D. thesis . Collage of Agriculture. Cairo Univ.

Felix, j. G; and B. Nina (2000). Use of the Minolta SPAD- 502 to determine chlorophyll concentration in ficus benjamina L. and populus deltoid's Marsh leaf tissue . Hort. Science. 35 (3): 423.

Gabr, M.A.; E.S.Elbana and A.M. Hussien (2006). Effect of summer pruning date and severity on vegetative growth, yield and fruit quality of Canino apricot trees. Alexandria J. Agri. Res. 51 (2) : 147 - 156 .

Hasani, G.H. and R. Rezaei (2007). Effect of training system and rate of pruning on yield and quality of peach fruit. J. Agri. Sci.( Univ. of Tabriz). 17 (1): 31-37.

Hossain , A.B. M. S. and F. Mizutani (2008). Dwarfing peach trees and fruit quality development by using summer pruning as physiological changed dwarfing Component . Australian J. Basic and Applied sci. 2 (4): 844-849.

Janick, J(2005). The origin of fruits, Fruit growing and Fruit breeding. Plant Breeding. Rev.25: 255-320.

Majed, S.M (2006). Effect of Summer Pruning and Paclobutrazol Spray on "ElAmar" Apricot Trees. M. Sc. Thesis, coll. Of Agric. Cairo Univ.

Punia, M.S (2007). Wild Apricot. National Oil Seeds and Vegetable Oils Development Board. Ministry of Agriculture, Govt. of India.

Said, L.A; F.M. Eissa and E.A. Kandil (2003). Effect of winter pruning, hand thinning and girdling on Canino apricot growth, yield and quality. Minia J. Agric. Res and Develop. 23(2):301-328. 


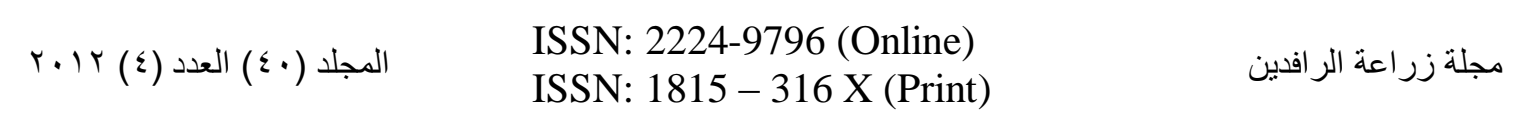

Son, L. and A. Kuden (2002). Effect of pruning treatment on the yield and fruit quality of precoce de tyrinthe apricot cultivar. Turk. J. Agric. For. 26(2):7986.

Sosna , I (2010). Effect of pruning time on growth, blooming and content of chemical constituents in leaves of four early ripening plum cultivars. J. Fruit and Ornamental Plant Res. 18 (2): 151-160.

Sosna, I (2010). Effect of pruning time on yielding and fruit quality of several early ripening plum cultivars. Acta Sci. Pol., Hortorum Cultus. 9(1): 37-44.

Wample, R.L; S.E. Spayed; R.G. Evans and R.G. Stevence (1991). Nitrogen fertilization and factors influencing grape vine cold hardiness. Inter . Symposium on Nitrogen in Grapes and Wine .120-125. Seattle. 18-19 June (Amer). Enol. Vitic. Davis, U.S.A.

Watanabe, M; A. Suzuki; S. Komori and H. Bessho (2006). Effect of heading back pruning on shoot growth and IAA and cytokinin concentrations at bud burst of columnar-type apple trees. J. Japanese . Soc. Hort. Sci. 75(3): 224-230.

Westwood , M.M (1993). Temperate - Zone Pomology, Physiological Culture . 3rd ed. Timber press. Part and Ore. From J. Amer. 33(4). 1998. 